MATHEMATICS OF COMPUTATION

Volume 75, Number 256, October 2006, Pages 1599-1616

S 0025-5718(06)01819-9

Article electronically published on June 7, 2006

\title{
A POSTERIORI FE ERROR CONTROL FOR P-LAPLACIAN BY GRADIENT RECOVERY IN QUASI-NORM
}

\author{
CARSTEN CARSTENSEN, W. LIU, AND N. YAN
}

\begin{abstract}
A posteriori error estimators based on quasi-norm gradient recovery are established for the finite element approximation of the p-Laplacian on unstructured meshes. The new a posteriori error estimators provide both upper and lower bounds in the quasi-norm for the discretization error. The main tools for the proofs of reliability are approximation error estimates for a local approximation operator in the quasi-norm.
\end{abstract}

\section{INTRODUCTION}

In this work we introduce a class of a posteriori error estimators for the finite element approximation of the p-Laplacian with homogeneous Dirichlet data

$$
-\operatorname{div}\left(|\nabla u|^{p-2} \nabla u\right)=f \text { in } \Omega \quad \text { and } \quad u=0 \text { on } \partial \Omega .
$$

Here, $1<p<\infty, \Omega$ is a bounded open subset of $\mathbb{R}^{2}$ with a Lipschitz boundary $\partial \Omega$, and $f$ is a given right-hand side. This equation is viewed as one of the typical examples of a large class of nonlinear problems. Indeed it is believed that essential difficulties in studies of finite element approximations for nonlinear systems are contained in (1.1) where many techniques, e.g., the linearization or deformation procedure, do not seem to work well.

Finite element approximations of the p-Laplacian have been studied extensively in the literature $\mathrm{Ci}, \mathrm{GM}, \mathrm{Ch}$. The quasi-norm approach for sharp a priori error bounds is summarized in [LY1, LY2]. An important aspect is the a posteriori error estimation of the p-Laplacian. In the contributions ODSD, BA, BL2, , P, V1 there are gaps in the power between the established upper and lower estimates. Recently LY1, LY2, the quasi-norm techniques and improved a posteriori error estimates of residual type were derived for the p-Laplacian. Initial analysis and numerical tests indicate that the new estimators are sharper than the very different existing ones, and, indeed, lead to more efficient computational meshes [CK, LY1].

In engineering simulations, a posteriori error estimators based on gradient recovery are widely used; see [AO, V2] for an introduction and [Ca, CB, CF1, CF2] for their mathematical justification. There seems to be no difficulty in designing and

Received by the editor April 16, 2003 and, in revised form, May 3, 2005.

2000 Mathematics Subject Classification. Primary 65N30, 49J40.

Key words and phrases. Finite element approximation, p-Laplacian, a posteriori error estimators, gradient recovery, quasi-norm error bounds.

Supported by the DFG Research Center MATHEON "Mathematics for key technologies" in Berlin.

(C)2006 American Mathematical Society 
using such estimators for the p-Laplacian using the standard Sobolev semi-norms, but, their mathematical justification was found difficult [CK].

The quasi-norm was used in LY3 to construct a posteriori error estimators based on gradient recovery, but the construction approach therein is restricted to uniform meshes. This paper aims at a justification for unstructured and locally refined meshes with different mathematical ideas. We derive upper and lower error bounds for the estimators and establish several approximation error estimates in the quasi-norms for the operator $\pi$ from Ca] (see Definition 4.2 below).

Efficient and reliable averaging techniques are very popular tools in practice. But they are also of particular importance for the understanding of a posteriori error estimation because they show that polution is excluded in the sense that the local approximation error dominates the global error.

The plan of this paper is as follows. In Section 2 we state some important inequalities and give the p-Laplacian its variational formulation. In Section 3 we set up the finite element approximation for the equation. We also introduce some quasi-norms and related results. In Section 4, we introduce the weighted Clementtype interpolator and we prove quasi-norm estimates for the interpolation error. In Section 5, we construct a posteriori error estimators based on quasi-norm gradient recovery on unstructured meshes, and we prove upper and lower error bounds for these estimators. Numerical experiments $[\mathrm{CK}]$ proved that the averaging estimators were surprisingly accurate in practice - quite in agreement with linear situations in CB, CF1, CF2 - provided the error measure is the quasi-norm.

\section{Preliminaries}

Throughout this paper we adopt the standard notation $W^{m, q}(\Omega)$ for Sobolev spaces on $\Omega$ with norm $\|\cdot\|_{W^{m, q}(\Omega)}$ and semi-norm $|\cdot|_{W^{m, q}(\Omega)}$. We set $W_{0}^{m, q}(\Omega) \equiv$ $\left\{w \in W^{m, q}(\Omega):\left.w\right|_{\partial \Omega}=0\right\}$. We denote $W^{m, 2}(\Omega)$ by $H^{m}(\Omega)$. In addition $c$ or $C$ denotes a general positive constant independent of $h$ and $A \leq C B$ abbreviates $A \lesssim B$.

The generic constant $C$ is only allowed to depend on $p, \Omega$, and the aspect ratio of the finite elements or the polynomial degree of piecewise polynomials under consideration.

The trace theorem [A, $\mathrm{BS}$ ] for $v \in W^{1, q}(\Omega)$ and $1 \leq q \leq \infty$ reads

$$
\|v\|_{L^{q}(\partial \Omega)} \lesssim\|v\|_{L^{q}(\Omega)}+|v|_{W^{1, q}(\Omega)} .
$$

For a triangle $K \in \mathcal{T}^{h}$ and for all $v \in W^{1, q}(K)$, this is

$$
\|v\|_{L^{q}(\partial K)} \lesssim h_{K}^{-\frac{1}{q}}\|v\|_{L^{q}(K)}+h_{K}^{1-\frac{1}{q}}|v|_{W^{1, q}(K)} .
$$

We need a quasi-norm version of the trace theorem for polynomials.

Lemma 2.1 (Lemma 3.6 in [LY2]). Let $K \in T^{h}$ and let $v$ be a polynomial of degree $\leq k$. Then

$$
h_{K} \int_{\partial K}\left(\left|\nabla u_{h}\right|+|\nabla v|\right)^{p-2}|\nabla v|^{2} d x \lesssim \int_{K}\left(\left|\nabla u_{h}\right|+|\nabla v|\right)^{p-2}|\nabla v|^{2} d x .
$$

The generic constant depends only on $k$ and the aspect ratio of the finite elements.

A display of elemetary (but sometimes tricky) estimates in $\mathbb{R}^{n}$ that play an essential role in our error analysis concludes this section. 
Lemma 2.2 (Main tools for quasi-norm from [LY1, BL3]).

(A) For all $a, \xi, \eta \geq 0, \theta>0$, it holds that

$$
(a+\xi)^{p-2} \xi \eta \leq \theta^{-\gamma}(a+\xi)^{p-2} \xi^{2}+\theta(a+\eta)^{p-2} \eta^{2},
$$

where

$$
\gamma= \begin{cases}1 & \text { if } 1<p \leq 2, \theta \in[1, \infty) \text { or if } 2<p<\infty, \theta \in(0,1), \\ \frac{1}{p-1} \quad \text { if } 1<p \leq 2, \theta \in(0,1) \text { or if } 2<p<\infty, \theta \in[1, \infty) .\end{cases}
$$

(B) For all $a, \xi, \eta \geq 0$, and $\delta>0$, it holds that

$$
\xi \eta \leq \delta^{-\beta}\left(a^{p-1}+\xi\right)^{p^{\prime}-2} \xi^{2}+\delta(a+\eta)^{p-2} \eta^{2},
$$

where $\beta$ is such that $\delta^{-\beta}=\max \left\{\delta^{-1}, \delta^{1 /(1-p)}\right\}$ and $1 / p+1 / p^{\prime}=1$.

(C) For all $\xi, \eta \in \mathbb{R}^{n}$ and $a \geq 0$, it holds that

$$
(a+|\xi+\eta|)^{p-2}|\xi+\eta|^{2} \lesssim(a+|\xi|)^{p-2}|\xi|^{2}+(a+|\eta|)^{p-2}|\eta|^{2} .
$$

The generic constant depends only on $1<p<\infty$.

\section{Finite element approximation of the P-Laplacian}

In this section we consider the finite element approximation of (WP) and introduce some quasi-norms. Given $f \in L^{2}(\Omega)$, the weak formulation of the p-Laplacian reads (WP) seek $u \in W_{0}^{1, p}(\Omega)$ with

$$
a(u, v)=(f, v) \quad \text { for all } v \in W_{0}^{1, p}(\Omega) .
$$

Here and throughout this paper,

$$
a(u, v)=\int_{\Omega}|\nabla u|^{p-2} \nabla u \cdot \nabla v d x \quad \text { and } \quad(w, v)=\int_{\Omega} w v d x .
$$

It is well established that there exists a unique solution to (WP).

Let $T^{h}$ be a regular triangulation [Ci, BS] of $\Omega$ into disjoint open regular triangles $K$, so that $\bar{\Omega}=\bigcup_{K \in T^{h}} \bar{K}$. Each element has at most one edge on $\partial \Omega$, and $\bar{K}$ and $\bar{K}^{\prime}$ have either only one common vertex or a whole edge if $K$ and $K^{\prime} \in T^{h}$. Let $h_{K}$ denote the maximum diameter of the element $K$ in $T^{h}$ and let $\rho_{K}$ denote the diameter of the largest ball contained in $K$. We assume that there is a regularity constant $R$ of $T^{h}$, independent of $h$, such that $1 \leq \max _{K \in T^{h}}\left(h_{K} / \rho_{K}\right) \leq R$. Let $h=\max _{K \in T^{h}} h_{K}$.

Associated with $T^{h}$ is a finite dimensional subspace $V^{h}$ of $C\left(\Omega^{h}\right)$, such that $\left.\chi\right|_{K}$ are linear functions for all $\chi \in V^{h}$ and $K \in T^{h}$ and

$$
V_{0}^{h}=\left\{v \in V^{h}: v=0 \text { on } \partial \Omega\right\} .
$$

The weak formula of the finite element approximation for (3.1) reads $\left(\mathrm{WP}^{h}\right)$ seek $u_{h} \in V_{0}^{h}$ with

$$
a\left(u_{h}, v_{h}\right)=\left(f, v_{h}\right) \quad \text { for all } v_{h} \in V_{0}^{h} .
$$

One of the key ideas in our approach is to introduce some quasi-norms to handle the degeneracy of the p-Laplacian in order to obtain sharp error bounds. We briefly introduce a quasi-norm and some relations between it and the standard Sobolev norms. Given $v, w \in W^{1, p}(\Omega)$, set

$$
|v|_{(w, p)}^{2} \equiv \int_{\Omega}|\nabla v|^{2}(|\nabla w|+|\nabla v|)^{(p-2)} d x .
$$


Proposition 3.1. $\quad$ (i) It holds that $|v|_{(w, p)} \geq 0$, and, when $v \in W_{0}^{1, p}(\Omega)$, $|v|_{(w, p)}=0$ if and only if $v=0$.

(ii) It holds that $\left|v_{1}+v_{2}\right|_{(w, p)} \lesssim\left|v_{1}\right|_{(w, p)}+\left|v_{2}\right|_{(w, p)}$ for any $v_{1}, v_{2} \in W^{1, p}(\Omega)$.

(iii) Furthermore, for $1<p \leq 2$, it holds that

$$
|v|_{W^{1, p}(\Omega)} \lesssim\left(|w|_{W^{1, p}(\Omega)},|v|_{W^{1, p}(\Omega)}\right)|v|_{(w, p)} \text { and }|v|_{(w, p)}^{2} \leq|v|_{W^{1, p}(\Omega)}^{p} .
$$

(iv) For $2 \leq p<\infty, s \in[2, p], r=s(2-p) /(2-s)$, it holds that

$$
|v|_{W^{1, p}(\Omega)}^{p} \leq|v|_{(w, p)}^{2} \leq C\left(|w|_{W^{1, r}(\Omega)},|v|_{W^{1, r}(\Omega)}\right)|v|_{W^{1, s}(\Omega)}^{2} .
$$

Proof. The conclusion (ii) can be proved with Lemma 2.2(C). The rest of the proposition can be shown as in BL3.

Remark 3.1. Throughout an a priori error analysis, $w$ is chosen to be $u$, the solution of (WP). To ensure the computability of the a posteriori error estimators it is replaced $u_{h}$ (or some postprocessed approximation of $u$ ) [LY1, LY2. A triangle inequality yields

$$
\left|u-u_{h}\right|_{(u, p)} \lesssim\left|u-u_{h}\right|_{\left(u_{h}, p\right)} \lesssim\left|u-u_{h}\right|_{(u, p)} .
$$

We shall simply write $|\cdot|_{(u, p)}$ as $|\cdot|_{(p)}$ when no confusion is likely to be caused.

Remark 3.2. The essential relations between the quasi-norm and the equation are reflected in the following inequalities. If $u$ solves (WP) and $v \in W^{1, p}(\Omega)$, then

$$
|u-v|_{(u, p)}^{2} \lesssim a(u, u-v)-a(v, u-v) .
$$

For any $\theta>0, v, w \in W^{1, p}(\Omega)$, and $\gamma$ from Lemma 2.2(A),

$$
|a(u, w)-a(v, w)| \lesssim \theta^{\gamma}|u-v|_{(u, p)}^{2}+\theta|w|_{(u, p)}^{2} .
$$

It follows from (3.6) - (3.7) that, for any $u, v \in W^{1, p}(\Omega)$,

$$
a(u, v-u)-a(v, u-v) \lesssim|u-v|_{(u, p)}^{2} \lesssim a(u, u-v)-a(v, u-v) .
$$

Thus the quasi-norm is naturally related to the total energy difference.

Remark 3.3. The relations (3.6)-(3.7) are important to prove the optimal a priori error bound in the quasi-norm BL1, LB5]

$$
\left|u-u_{h}\right|_{(p)}^{2} \lesssim \min _{v_{h} \in V_{0}^{h}}\left|u-v_{h}\right|_{(p)}^{2} \lesssim h^{2}
$$

when $u$ is smooth enough, where

$$
|u-v|_{(p)}^{2}=|u-v|_{(u, p)}^{2}=\int_{\Omega}(|\nabla u|+|\nabla(u-v)|)^{p-2}|\nabla(u-v)|^{2} d x .
$$

Thus when $1<p \leq 2$, one has the optimal a priori error bound in $W^{1, p}$,

$$
\left\|u-u_{h}\right\|_{W^{1, p}(\Omega)} \lesssim\left|u-u_{h}\right|_{(p)} \lesssim h .
$$

Remark 3.4. In [LY1, LY2, the quasi-norm has been used to derive improved a posteriori error estimates for the p-Laplacian. For instance, let $u_{h}$ be the finite element approximation of (3.1) and let $1 / p+1 / p^{\prime}=1$. Then

$$
\eta_{1}^{2}+\eta_{2}^{2}+\epsilon_{1} \lesssim\left|u-u_{h}\right|_{(p)}^{2} \lesssim \eta_{1}^{2}+\eta_{2}^{2}+\epsilon_{2}
$$


with higher order terms $\varepsilon_{1}$ and $\varepsilon_{2}$ and

$$
\begin{aligned}
& \eta_{1}^{2}=\sum_{K} \int_{K}\left(\left|\nabla u_{h}\right|^{p-1}+h_{K}|f|\right)^{p^{\prime}-2} h_{K}^{2}|f|^{2} d x, \\
& \eta_{2}^{2}=\sum_{l} \int_{K_{l}}\left(\left|\nabla u_{h}\right|^{p-1}+\left|A_{l}\right|\right)^{p^{\prime}-2} A_{l}^{2} d x, \\
& A_{l}=\left(\left(\left|\nabla u_{h}\right|^{p-2} \nabla u_{h}\right)_{K_{l}^{1}}-\left(\left|\nabla u_{h}\right|^{p-2} \nabla u_{h}\right)_{K_{l}^{2}}\right) n,
\end{aligned}
$$

where $n$ is the unit normal vector on $l=\bar{K}_{l}^{1} \cap \bar{K}_{l}^{2}$ outward $K_{l}^{1}$.

\section{NEW RESULTS ON QUASI-NORM APPROXIMATION}

This section establishes essential approximation error estimates in the quasinorm. Here we take a general approach so that the arguments can be applied to a more general class of degenerate systems of Remark 4.3 .

Definition 4.1. For $x, y \geq 0$ and $1<p<\infty$, let

$$
G(x, y):= \begin{cases}y^{2}(x+y)^{p-2} & \text { if } x+y>0 \\ 0 & \text { if } x=y=0 .\end{cases}
$$

Remark 4.1. Without further (explicit) notice, we shall use that $G(x, y)$ is monotone increasing and convex with respect to the variable $y$.

First, we prove a quasi-norm version of the quotient theorem.

Lemma 4.1. Let $\Omega$ be a nonempty bounded convex open set in $\mathbb{R}^{2}$. Let $1<p<\infty$ and $f \in\left(W^{1, p}(\Omega)\right)^{*}$ with $\mathbb{R} \cap \operatorname{Ker} f=\{0\}$, where $\mathbb{R}$ is the space of functions constant on the domain $\Omega$. Then there exists a constant $c_{1}=c(f, p, \Omega)$ such that, for all $a \in R, a \geq 0$, and $v \in W^{1, p}(\Omega)$,

$$
\int_{\Omega} G(a,|v|) d x \leq c_{1} G(a,|f(v)|)+c_{1} \int_{\Omega} G(a,|\nabla v|) d x .
$$

Proof. We argue by contradiction and suppose that the lemma is false. Then there would exist a sequence $v_{j}$ in $W^{1, p}(\Omega)$ with $\delta_{j}:=\left\|v_{j}\right\|_{W^{1, q}(\Omega)}>0, q=\min \{2, p\}$, and a sequence $a_{j}$ of nonnegative real numbers such that

$$
G\left(a_{j},\left|f\left(v_{j}\right)\right|\right)+\int_{\Omega} G\left(a_{j},\left|\nabla v_{j}\right|\right) d x \leq 1 / j \int_{\Omega} G\left(a_{j},\left|v_{j}\right|\right) d x
$$

for all $j \in \mathbb{N}$. We observe in any case there exists a $u \in W^{1, q}(\Omega)$ with

$$
u_{j}:=v_{j} / \delta_{j} \text { satisfies }\left\|u_{j}\right\|_{W^{1, q}(\Omega)}=1, \quad u_{j} \rightarrow u \text { in } W^{1, q}(\Omega) .
$$

Here we have chosen a weak convergent subsequence with Banach Alaoglu's theorem. In the first case we suppose that there exists a constant $\gamma, 0<\gamma<\infty$, with

$$
a_{j} \leq \gamma \delta_{j} \quad \text { for } j=1,2,3, \ldots
$$

At least we suppose (4.3) for a subsequence we have not relabelled. If $1<p \leq 2$, then $G(a, x) \leq x^{p}$ for all $x \geq 0$. Therefore, even without (4.3),

$$
\int_{\Omega} G\left(a_{j} / \delta_{j},\left|u_{j}\right|\right) d x \leq\left\|u_{j}\right\|_{L^{p}(\Omega)}^{p} \leq 1 .
$$


If $2 \leq p$, then $G\left(\cdot,\left|u_{j}\right|\right)$ is monotone increasing. Hence, (4.2)-(4.3) yields

$$
\begin{aligned}
\int_{\Omega} G\left(a_{j} / \delta_{j},\left|u_{j}\right|\right) d x & \leq \int_{\Omega}\left(\gamma+\left|u_{j}\right|\right)^{p-2}\left|u_{j}\right|^{2} d x \\
& \leq\left\|\gamma+\left|u_{j}\right|\right\|_{L^{p}(\Omega)}^{p} \leq\left(1+\gamma|\Omega|^{1 / p}\right)^{p} .
\end{aligned}
$$

Hence, for all $1<p<\infty, \int_{\Omega} G\left(a_{j} / \delta_{j},\left|u_{j}\right|\right) d x$ is bounded. A scaling of (4.1) then shows

$$
\lim _{j \rightarrow \infty} \int_{\Omega} G\left(a_{j} / \delta_{j},\left|\nabla u_{j}\right|\right) d x=\lim _{j \rightarrow \infty} G\left(a_{j} / \delta_{j},\left|f\left(u_{j}\right)\right|\right)=0 .
$$

If $1<p \leq 2$, a Hölder inequality with exponents $2 / p$ and $2 /(2-p)$ leads to

$$
\begin{aligned}
\left\|\nabla u_{j}\right\|_{L^{p}(\Omega)}^{p} & =\int_{\Omega}\left|\nabla u_{j}\right|^{p}\left(a_{j} / \delta_{j}+\left|\nabla u_{j}\right|\right)^{p(p-2) / 2}\left(a_{j} / \delta_{j}+\left|\nabla u_{j}\right|\right)^{p(2-p) / 2} d x \\
& \leq\left(\int_{\Omega} G\left(a_{j} / \delta_{j},\left|\nabla u_{j}\right|\right) d x\right)^{p / 2}\left(\int_{\Omega}\left(a_{j} / \delta_{j}+\left|\nabla u_{j}\right|\right)^{p} d x\right)^{1-p / 2} .
\end{aligned}
$$

The last factor is bounded as $j \rightarrow \infty$ by (4.2)- (4.3) and the second to last factor tends to zero by (4.5). Again, for $1<p \leq 2$ (when $G\left(\cdot,\left|f\left(u_{j}\right)\right|\right.$ ) is monotone decreasing), (4.5) shows that $G\left(\gamma,\left|f\left(u_{j}\right)\right|\right)$ tends to zero and, hence, so does $\left|f\left(u_{j}\right)\right|$. Consequently,

$$
\lim _{j \rightarrow \infty}\left\|\nabla u_{j}\right\|_{L^{p}(\Omega)}=\lim _{j \rightarrow \infty}\left|f\left(u_{j}\right)\right|=0 .
$$

So far we established (4.7) for $1<p \leq 2$. For $2<p<\infty,\left|\nabla u_{j}\right|^{p} \leq G\left(a_{j} / \delta_{j},\left|\nabla u_{j}\right|\right)$ and $\left|f\left(u_{j}\right)\right|^{p} \leq G\left(a_{j} / \delta_{j},\left|f\left(u_{j}\right)\right|\right)$ and so (4.5) implies (4.7) directly. From (4.7) we deduce a contradiction to (4.2): Since $W^{1, q}(\Omega)$ is compactly embedded in $L^{q}(\Omega)$ we have $u_{j} \rightarrow u$ in $L^{q}(\Omega)$. With (4.7), $u_{j} \rightarrow u$ in $W^{1, q}(\Omega)$ and so $\|u\|_{W^{1, q}(\Omega)}=1$. Conversely, $u$ is constant (as $\nabla u_{j} \rightarrow 0$ in $L^{q}(\Omega)$ ). Since $f$ is a bounded linear form, $f\left(u_{j}\right) \rightarrow f(u)$ and $f(u)=0$. Since $u \in P_{0}(\Omega) \cap \operatorname{Ker} f$, we have $u=0$. This contradiction with $\|u\|_{W^{1, q}(\Omega)}=1$ concludes the proof in case (4.3).

In the remaining second case we suppose that $a_{j} / \delta_{j}$ is not bounded (even not for a subsequence). Hence, $\lim _{j \rightarrow \infty} a_{j} / \delta_{j}=+\infty$. One can assume that

$$
\delta_{j} \leq \gamma a_{j} \text { for } q=\min \{2, p\} \text { and for } j=1,2,3, \ldots
$$

for a constant $\gamma$ (and at least for sufficiently large $j$ which we have not relabelled). If $1<p \leq 2$, we use $\left(1+\delta_{j} / a_{j}\left|u_{j}\right|\right)^{p-2} \leq 1$. If $2 \leq p<\infty$, we use $\delta_{j} / a_{j} \leq \gamma$. This leads to

$$
\begin{aligned}
1 / j \int_{\Omega}\left(1+\delta_{j} / a_{j}\left|u_{j}\right|\right)^{p-2} \mid & \left.u_{j}\right|^{2} d x \\
\leq & \begin{cases}\left\|u_{j}\right\|_{L^{2}(\Omega)}^{2} / j & \text { if } 1<p \leq 2, \\
\left(\left\|u_{j}\right\|_{L^{p}(\Omega)}+\gamma|\Omega|^{1 / p}\right)^{p} / j & \text { if } 2 \leq p<\infty .\end{cases}
\end{aligned}
$$

Since $q=\min \{2, p\}$ and $\left\|u_{j}\right\|_{W^{1, q}(\Omega)}=1$, we conclude that (4.9) tends to zero as $j \rightarrow \infty$ from embedding. Therefore, a scaling of (4.1) yields

$$
\begin{aligned}
\lim _{j \rightarrow \infty} \int_{\Omega}\left(1+\delta_{j} / a_{j}\left|\nabla u_{j}\right|\right)^{p-2} \mid \nabla & \left.u_{j}\right|^{2} d x \\
& =\lim _{j \rightarrow \infty}\left(1+\delta_{j} / a_{j}\left|f\left(u_{j}\right)\right|\right)^{p-2}\left|f\left(u_{j}\right)\right|^{2}=0 .
\end{aligned}
$$


If $2 \leq p<\infty$, we directly deduce (4.7) for $q=2$ and finish the proof as in the first case since $\left\|u_{j}\right\|_{H^{1}(\Omega)}=1$. If $1<p \leq 2$, we argue with a Hölder inequality analogy to (4.6) and infer

$$
\left\|\nabla u_{j}\right\|_{L^{p}(\Omega)}^{2} \leq \int_{\Omega}\left(1+\delta_{j} / a_{j}\left|\nabla u_{j}\right|\right)^{p-2}\left|\nabla u_{j}\right|^{2} d x\left(\int_{\Omega}\left(1+\delta_{j} / a_{j}\left|\nabla u_{j}\right|\right)^{p} d x\right)^{2-p} .
$$

The last factor is bounded according to (4.8) and $\left\|u_{j}\right\|_{W^{1, p}(\Omega)}=1$. This and (4.10) show (4.7) with $p=q \leq 2$. The proof is then finished as in the first case.

Remark 4.2. Lemma 4.1 is employed in connection with a scaling argument. If we scale the domain $\Omega$ from a reference size 1 to a patch-size $h$, the first term obtains the factor $h^{2}$ from a change of variables while the last term in values $a h|\nabla v|$ instead of $|\nabla v|$. With a different $a$, this yields

$$
\int_{\Omega} G(a, v) d x \lesssim \int_{\Omega} G(a, h|\nabla v|) d x
$$

for all $v \in W^{1, p}(\Omega) \cap \operatorname{Ker}(f)$ and $h=\operatorname{diam}(\Omega)$; the generic constant depends on the shape of $\Omega$ but is $h$-independent.

Recal a weighted Clement-type interpolation on the finite element space $V_{0}^{h}$.

Definition 4.2 ( $\mathrm{Ca}$ ). Let $D$ be the set of nodes,

$$
\Lambda=\{z \in D: z \cap \partial \Omega=\emptyset\} .
$$

Given the nodal basis function $\varphi_{z}$ of $z$ in $V^{h}$, set $\omega_{z}=\left\{x \subset \Omega: \varphi_{z}(x)>0\right\}$,

$$
\psi_{z}=\varphi_{z} / \psi \quad \text { and } \quad \psi=\sum_{z \in \Lambda} \varphi_{z} .
$$

Then, for all $v \in W_{0}^{1, p}(\Omega)$, define the interpolation of $v$ by

$$
\pi v=\sum_{z \in \Lambda} v_{z} \varphi_{z} \in V_{0}^{h}, \quad v_{z}=\int_{\Omega} \psi_{z} v d x / \int_{\Omega} \varphi_{z} d x
$$

It is essential for later analysis to establish approximation error estimates in the quasi-norm for the operator $\pi$.

Lemma 4.2. For any $1<p<\infty$ and positive integers $d$ and $n$ there exists a constant $c_{2}=c(p, d, n)$ such that, for all $a_{1}, a_{2}, \ldots, a_{n} \in \mathbb{R}^{d}$, it holds that

$$
\sum_{j=1}^{n} \sum_{k=1}^{j-1} G\left(\left|a_{j}\right|,\left|a_{j}-a_{k}\right|\right) \leq c_{2} \sum_{\ell=1}^{n-1} \min _{m=1, \ldots, n} G\left(\left|a_{m}\right|,\left|a_{\ell+1}-a_{\ell}\right|\right)
$$

Proof. Let $\alpha:=\left(a_{1}+\cdots+a_{n}\right) / n \in \mathbb{R}^{d}$ and $b_{j}:=a_{j}-\alpha \in \mathbb{R}^{d}$ so that $b_{1}+\cdots+b_{n}=0$. Define

$$
\begin{aligned}
f\left(\alpha ; b_{1}, \ldots, b_{n}\right) & :=\sum_{j=1}^{n} \sum_{k=1}^{j-1} G\left(\left|\alpha+b_{j}\right|,\left|b_{j}-b_{k}\right|\right), \\
g\left(\alpha ; b_{1}, \ldots, b_{n}\right) & :=\sum_{\ell=1}^{n-1} \min _{m=1, \ldots, n} G\left(\left|\alpha+b_{m}\right|,\left|b_{\ell+1}-b_{\ell}\right|\right) .
\end{aligned}
$$

Observe that $g(\alpha, \cdot)$ is positive for nonzero arguments on

$$
X:=\left\{\left(b_{1}, \ldots, b_{n}\right) \in \mathbb{R}^{d \times n}: b_{1}+\cdots+b_{n}=0\right\},
$$


since $g\left(\alpha ; b_{1}, \ldots, b_{n}\right)=0$ implies $b_{1}=b_{2}=\cdots=b_{n}$. Let $B:=\left\{\left(b_{1}, \ldots, b_{n}\right) \in X\right.$ : $\left.\left|b_{1}\right|^{2}+\cdots+\left|b_{n}\right|^{2}=1\right\}$ denote the unit ball surface in $X$. Then, for any $\beta \in \mathbb{R}^{d}$,

$$
c(\beta):=\max _{\left(b_{1}, \ldots, b_{n}\right) \in B} f\left(\beta ; b_{1}, \ldots, b_{n}\right) / g\left(\beta ; b_{1}, \ldots, b_{n}\right)<\infty,
$$

since the denominator is positive and $f(\alpha ; \cdot), g(\alpha ; \cdot)$ are continuous on the compact set $B$. The same argument shows

$$
c_{\infty}:=\max _{\left(b_{1}, \ldots, b_{n}\right) \in X \backslash\{0\}} \sum_{j=1}^{n} \sum_{k=1}^{j-1}\left|b_{j}-b_{k}\right|^{2} / \sum_{\ell=1}^{n-1}\left|b_{\ell+1}-b_{\ell}\right|^{2}<\infty .
$$

Note that $\lim \sup _{|\beta| \rightarrow \infty} c(\beta) \leq c_{\infty}<\infty$ and so $c(\beta)$ is a bounded continuous function in $\beta \in \mathbb{R}^{d}$. For all $a_{1}, \ldots, a_{n} \in \mathbb{R}^{d}$, we have $\alpha \in \mathbb{R}^{d}$, and $\left(b_{1}, \ldots, b_{n}\right) \in X$ as above. Since $f$ and $g$ are positively homogeneous functions we have, for $\lambda:=$ $\left(\left|b_{1}\right|^{2}+\cdots+\left|b_{n}\right|^{2}\right)^{1 / 2}>0$

$$
\begin{aligned}
f\left(\alpha ; b_{1}, \ldots, b_{n}\right) & =\lambda f\left(\alpha / \lambda ; b_{1} / \lambda, \ldots, b_{n} / \lambda\right) \\
& \lesssim \lambda g\left(\alpha / \lambda, b_{1} / \lambda, \ldots, b_{n} / \lambda\right) \\
& \lesssim g\left(\alpha ; b_{1}, \ldots, b_{n}\right) .
\end{aligned}
$$

Let $[w]_{l}:=\left.w\right|_{K_{l}^{1}}-\left.w\right|_{K_{l}^{2}}$ along $l=\bar{K}_{l}^{1} \cap \bar{K}_{l}^{2}$. Then, we have the following interpolation error estimates for the operator $\pi$ in the quasi-norm.

Lemma 4.3. Let $\pi$ be the operator of Definition 4.2, $1<p<\infty, u_{h} \in V_{h}$, $v \in W_{0}^{1, p}(\Omega)$, and $K \in T^{h}$. Set $\mathcal{T}_{z}:=\left\{K \in T^{h}: K \subseteq \bar{\omega}_{z}\right\}, \bigcup \mathcal{E}_{z}:=\bigcup\{\partial K: K \in$ $\left.\mathcal{T}_{z}\right\}$, and let $\left[\partial u_{h} / \partial n_{\varepsilon}\right]$ denote the jump of the discrete normal fluxes across inner element boundaries. Then it holds that

$$
\begin{aligned}
& \int_{K} G\left(\left|\nabla u_{h}\right|,|v-\pi v| / h_{K}\right) d x+\int_{K} G\left(\left|\nabla u_{h}\right|,|\nabla(v-\pi v)|\right) d x \\
& \lesssim \sum_{z \in \Lambda \cap K}\left(\int_{\omega_{z}} G\left(\left|\nabla u_{h}\right|,|\nabla v|\right) d x+\min _{T \in \mathcal{T}_{z}} \int_{\cup \mathcal{E}_{z}} G\left(\left|\nabla u_{h}\right|_{T}|,|\left[\partial u_{h} / \partial n_{\mathcal{E}}\right] \mid\right) d s\right)
\end{aligned}
$$

and (where $\sum_{l \cap \partial \Omega=\emptyset}$ denotes a sum over all interior edges l)

$$
\begin{aligned}
& \sum_{K \in T^{h}} \int_{K} G\left(\left|\nabla u_{h}\right|,|v-\pi v| / h_{K}\right) d x+\int_{\Omega} G\left(\left|\nabla u_{h}\right|,|\nabla(v-\pi v)|\right) d x \\
& \lesssim \int_{\Omega} G\left(\left|\nabla u_{h}\right|,|\nabla v|\right) d x \\
&+\sum_{l \cap \partial \Omega=\emptyset} \min _{\bar{K} \cap \neq \emptyset} \int_{K}\left(\left|\nabla u_{h}\right|+\left|\left[\frac{\partial u_{h}}{\partial n}\right]_{l}\right|\right)^{p-2}\left|\left[\frac{\partial u_{h}}{\partial n}\right]_{l}\right|^{2} d x
\end{aligned}
$$

Proof. In the first step, we show that the first term of the left-hand side of (4.11) is bounded by the right-hand side. Fix $a:=\left.\nabla u_{h}\right|_{K}$ and set $v_{z}:=(\pi v)(z)$ for all $z \in D$. Since $\left(\psi_{z}\right)_{z \in \Lambda \cap K}$ is a partition of unity on $K$, and $G$ satisfies a triangle inequality in the sense of Lemma 2.2(C),

$$
\begin{aligned}
\int_{K} G\left(\left|\nabla u_{h}\right|,|v-\pi v| / h_{K}\right) d x & =\int_{K} G\left(|a|,\left|\sum_{z \in \Lambda \cap K}\left(v \psi_{z}-v_{z} \varphi_{z}\right)\right| / h_{K}\right) d x \\
& \lesssim \sum_{z \in \Lambda \cap K} \int_{K} G\left(|a|,\left|v \psi_{z}-v_{z} \varphi_{z}\right| / h_{K}\right) d x
\end{aligned}
$$


Since $K \subseteq \bar{\omega}_{z}$, we have, for any fixed $z \in \Lambda \cap K$,

$$
\int_{K} G\left(|a|,\left|v \psi_{z}-v_{z} \varphi_{z}\right| / h_{K}\right) d x \leq \int_{\omega_{z}} G\left(|a|,\left|v \psi_{z}-v_{z} \varphi_{z}\right| / h_{K}\right) d x
$$

In the first case, we suppose $\psi_{z}=\varphi_{z}$, i.e., all nodes in $\bar{\omega}_{z}$ are free nodes. A scaled version of Lemma 4.1 with $w:=\left(v-v_{z}\right) / h_{K}, f(w)=\int_{\omega_{z}} \varphi_{z} w d x$, and $\psi_{z}=\varphi_{z}$ and $\left|\varphi_{z}\right| \leq 1$ yield

$$
\begin{aligned}
\int_{\omega_{z}} G\left(|a|,\left|v \psi_{z}-v_{z} \varphi_{z}\right| / h_{K}\right) d x & \leq \int_{\omega_{z}} G\left(|a|,\left|v-v_{z}\right| / h_{K}\right) d x \\
& \lesssim \int_{\omega_{z}} G(|a|,|\nabla v|) d x .
\end{aligned}
$$

In the remaining second case, $\psi \not \equiv 1$ on $\omega_{z}$ and so, $\partial \omega_{z} \cap \partial \Omega$ includes at least one outer edge $E$. With $f(w)=\int_{E} w d x$, we deduce from Lemma 4.1 that

$$
\int_{\omega_{z}} G\left(|a|,\left|v \psi_{z}\right| / h_{K}\right) d x \leq \int_{\omega_{z}} G\left(|a|,|v| / h_{K}\right) d x \lesssim \int_{\omega_{z}} G(|a|,|\nabla v|) d x .
$$

Since $\left|v_{z}\right| \lesssim\left|f_{\omega_{z}} \psi_{z} v d x\right|$ and $G\left(|a|, \cdot / h_{K}\right)$ is convex, Jensen's inequality shows that

$$
\begin{aligned}
& \int_{\omega_{z}} G\left(|a|,\left|v_{z}\right| / h_{K}\right) d x \lesssim \int_{\omega_{z}} G\left(|a|,\left|\int_{\omega_{z}} \psi_{z} v d x\right| / h_{K}\right) d y \\
& \quad \leq \int_{\omega_{z}} f_{\omega_{z}} G\left(|a|,\left|\psi_{z} v\right| / h_{K}\right) d x d y=\int_{\omega_{z}} G\left(|a|,\left|\psi_{z} v\right| / h_{K}\right) d x .
\end{aligned}
$$

Here and throughout, the sign $f_{\omega_{z}}$ represents the integral average over $\omega_{z}$. The triangle inequality of Lemma $2.2(\mathrm{C}),(4.15)-(4.16)$, the monotonicity in the second argument of $G$, and the inequalities $0 \leq \varphi_{z} \leq 1,0 \leq \psi \leq 1$ prove that

$$
\begin{aligned}
\int_{\omega_{z}} G\left(|a|,\left|v \psi_{z}-v_{z} \varphi_{z}\right| / h_{K}\right) d x \lesssim \int_{\omega_{z}} G\left(|a|,\left|v \psi_{z}\right| / h_{K}\right) d x \\
+\int_{\omega_{z}} G\left(|a|,\left|v_{z} \varphi_{z}\right| / h_{K}\right) d x \lesssim \int_{\omega_{z}} G(|a|,|\nabla v|) d x .
\end{aligned}
$$

Notice that $a+\left|\nabla u_{h}-a\right| \approx a+\left|\nabla u_{h}\right| \approx\left|\nabla u_{h}\right|+\left|a-\nabla u_{h}\right|$ and so

$$
\begin{aligned}
G(|a|,|\nabla v|) & \leq G\left(|a|,|\nabla v|+\left|a-\nabla u_{h}\right|\right) \\
& =\left(|a|+\left|a-\nabla u_{h}\right|+|\nabla v|\right)^{p-2}\left(|\nabla v|+\left|a-\nabla u_{h}\right|\right)^{2} \\
& \lesssim\left(\left|\nabla u_{h}\right|+|\nabla v|+\left|a-\nabla u_{h}\right|\right)^{p-2}\left(|\nabla v|+\left|a-\nabla u_{h}\right|\right)^{2} \\
& =G\left(|a|,|\nabla v|+\left|a-\nabla u_{h}\right|\right) .
\end{aligned}
$$

The triangle inequality of Lemma 2.2(C) shows that

$$
G(|a|,|\nabla v|) \lesssim G\left(\left|\nabla u_{h}\right|,|\nabla v|\right)+G\left(\left|\nabla u_{h}\right|,\left|a-\nabla u_{h}\right|\right) d x
$$

This, (4.15), and (4.17) result in

$$
\begin{aligned}
\int_{\omega_{z}} G\left(|a|, \mid v \psi_{z}-\right. & \left.v_{z} \varphi_{z} \mid / h_{K}\right) d x \\
& \lesssim \int_{\omega_{z}} G\left(\left|\nabla u_{h}\right|,|\nabla v|\right) d x+\int_{\omega_{z}} G\left(\left|\nabla u_{h}\right|,\left|a-\nabla u_{h}\right|\right) d x .
\end{aligned}
$$

This and Lemma 4.2 with $a_{j}=\left.\nabla u_{h}\right|_{K_{j}}$ for $K_{1}, \ldots, K_{n} \in T^{h}$ with $K_{1} \cup \cdots \cup K_{n}=\bar{\omega}_{z}$ and $\left\{K_{1} \cap K_{2}, \ldots, K_{n-1} \cap K_{n}\right\}=\left\{E \in \mathcal{E}: E \subseteq \bar{\omega}_{z} \backslash \partial \Omega\right\}$ proves that (4.13) is 
bounded by the right-hand side of (4.11). The second step is to show that the second term on the left-hand side of (4.11) is bounded in this way as well. To this end, we let $c$ be the integral mean of $v$ on $K$. The triangle inequality of Lemma 2.2(C) shows that

$$
\begin{aligned}
\int_{K} G\left(\left|\nabla u_{h}\right|, \mid \nabla(v\right. & -\pi v) \mid) d x \\
& \lesssim \int_{K} G\left(\left|\nabla u_{h}\right|,|\nabla v|\right) d x+\int_{K} G\left(\left|\nabla u_{h}\right|,|\nabla(\pi v-c)|\right) d x .
\end{aligned}
$$

The first term on the right-hand side of (4.21) is already bounded as asserted. To estimate the second term, note that $\pi v-c$ is an affine function on $K$. Then an inverse estimate shows that

$$
|\nabla(\pi v-c)| \lesssim f_{K}|\pi v-c| d x / h_{K}
$$

( $f$ represents the integral average). Jensen's inequality leads to

$$
\begin{aligned}
\int_{K} G\left(\left|\nabla u_{h}\right|,|\nabla(\pi v-c)|\right) d x & \lesssim \int_{K} f_{K} G\left(\nabla u_{h}|,| \pi v-c \mid / h_{K}\right) d x d y \\
& =\int_{K} G\left(\left|\nabla u_{h}\right|,|\pi v-c| / h_{K}\right) d x
\end{aligned}
$$

The triangle inequality of Lemma 2.2(C) yields

$$
\begin{aligned}
\int_{K} G\left(\left|\nabla u_{h}\right|,|\pi v-c| / h_{K}\right) d x & \\
& \lesssim \int_{K} G\left(\left|\nabla u_{h}\right|,|v-\pi v| / h_{K}\right) d x+\int_{K} G\left(\left|\nabla u_{h}\right|,|v-c| / h_{K}\right) d x .
\end{aligned}
$$

The first term on the right-hand side of (4.23) is already shown to be bounded by the right-hand side of (4.11). The same conclusion for the second term follows from Lemma 4.1 with $f(w)=\int_{K} w d x$ and $\Omega=K$ as in (4.15):

$$
\int_{K} G\left(\left|\nabla u_{h}\right|,|v-c| / h_{K}\right) d x \lesssim \int_{K} G\left(\left|\nabla u_{h}\right|,|\nabla v|\right) d x .
$$

Then (4.21)-(4.24) imply that

$$
\begin{aligned}
\int_{K} G\left(\left|\nabla u_{h}\right|, \mid \nabla\right. & (v-\pi v) \mid) d x \\
& \lesssim \int_{K} G\left(\left|\nabla u_{h}\right|,|\nabla v|\right) d x+\int_{K} G\left(\left|\nabla u_{h}\right|,|v-\pi v| / h_{K}\right) d x .
\end{aligned}
$$

Hence the desired estimate of the second term on the left-hand side of (4.11) follows from (4.25) and the first step of the proof.

The next lemma establishes a quasi-norm estimate for the inner product of a function and an interpolation error. Let $h_{z}=\max _{K \subset \omega_{z}} h_{K}$ denote the maximal mesh-size in the patch $\omega_{z}$. 
Lemma 4.4. For any $\delta>0,1<p, p^{\prime}<\infty$ with $1 / p+1 / p^{\prime}=1, u_{h} \in V_{h}$, $v \in W_{0}^{1, p}(\Omega)$, and $f \in W^{1, p^{\prime}}(\Omega)$ it holds that

$$
\begin{aligned}
\int_{\Omega} f(v-\pi v) d x \leq & C \delta \int_{\Omega} G\left(\left|\nabla u_{h}\right|,|\nabla v|\right) d x \\
& +C(\delta) \sum_{z \in \Lambda} \int_{\omega_{z}}\left(\left|\nabla u_{h}\right|^{p-1}+h_{z}^{2}|\nabla f|\right)^{p^{\prime}-2} h_{z}^{4}|\nabla f|^{2} d x \\
& +C \delta \sum_{z \in \Lambda} \min _{K \in \mathcal{T}_{z}} \int_{\cup \mathcal{E}_{z}} G\left(\left|\nabla u_{h}\right|_{K} \mid,\left[\partial u_{h} / \partial n_{\varepsilon}\right]\right) d s .
\end{aligned}
$$

With $\tilde{\eta}$ of Lemma 4.3, it holds that

$$
\begin{aligned}
\int_{\Omega} f(v-\pi v) d x \leq & C(\delta) \sum_{K \in T^{h}} \int_{K}\left(\left|\nabla u_{h}\right|^{p-1}+h_{K}^{2}|\nabla f|\right)^{p^{\prime}-2} h_{K}^{4}|\nabla f|^{2} d x \\
& +C \delta \sum_{K \in T^{h}} \int_{K}\left(\left|\nabla u_{h}\right|+|\nabla v|\right)^{p-2}|\nabla v|^{2} d x+C \delta \tilde{\eta}^{2} .
\end{aligned}
$$

Proof. First note that $\int\left(v \psi_{z}-v_{z} \varphi_{z}\right) d x=0$. Thus, with the integral mean $f_{z}:=$ $f_{\omega_{z}} f(x) d x$

$$
\begin{aligned}
\int_{\Omega} f(v-\pi v) d x & =\sum_{z \in \Lambda} \int_{\Omega} f\left(v \psi_{z}-v_{z} \varphi_{z}\right) d x \\
& =\sum_{z \in \Lambda} \int_{\omega_{z}}\left(f-f_{z}\right) h_{z}\left(v \psi_{z}-v_{z} \varphi_{z}\right) / h_{z} d x .
\end{aligned}
$$

Lemma 2.2(B) allows an estimate of the product and so

$$
\begin{aligned}
& \int_{\omega_{z}}\left(f-f_{z}\right) h_{z}\left(v \psi_{z}-v_{z} \varphi_{z}\right) / h_{z} d x \\
\leq & \delta^{-\beta} \int_{\omega_{z}}\left(|a|^{p-1}+\left|f-f_{z}\right| h_{z}\right)^{p^{\prime}-2} h_{z}^{2}\left|f-f_{z}\right|^{2} d x \\
& \left.+\delta \int_{\omega_{z}} G\left(|a|, \mid v \psi_{z}-v_{z} \varphi_{z}\right) \mid / h_{z}\right) d x .
\end{aligned}
$$

Here $a$ is one of the discrete gradients $\left|\nabla u_{h}\right|$ on $\omega_{z}$. Lemma 4.1 will be employed for $f-f_{z}$ and the functional $g(w)=\int_{\omega_{z}} w$ (so it vanishes for $w:=f-f_{z}$ ). Notice that $a$ is replaced by $|a|^{p-1}$ and $p$ is replaced by $p^{\prime}$. Then we obtain

$$
\begin{aligned}
\int_{\omega_{z}}\left(|a|^{p-1}+\left|f-f_{z}\right| h_{z}\right)^{p^{\prime}-2} h_{z}^{2} \mid f & -\left.f_{z}\right|^{2} d x \\
& \lesssim \int_{\omega_{z}}\left(|a|^{p-1}+h_{z}^{2}|\nabla f|\right)^{p^{\prime}-2} h_{z}^{4}|\nabla f|^{2} d x .
\end{aligned}
$$

Arguing as in proving (4.14)-(4.17), we deduce from (4.29)-(4.30) that

$$
\begin{aligned}
\int_{\omega_{z}}(f & \left.-f_{z}\right) h_{z}\left(v \psi_{z}-v_{z} \varphi_{z}\right) / h_{z} d x \\
& \lesssim \delta \int_{\omega_{z}} G(|a|,|\nabla v|) d x+\delta^{-\beta} \int_{\omega_{z}}\left(|a|^{p-1}+h_{z}^{2}|\nabla f|\right)^{p^{\prime}-2} h_{z}^{4}|\nabla f|^{2} d x .
\end{aligned}
$$


So far, $a$ is a constant vector on $\omega_{z}$. Depending on $p^{\prime}$, we choose $a$ so that $|a|$ is minimal or maximal amongst $\left(\left|\nabla u_{h}\right|_{K}: K \in \mathcal{T}_{z}\right)$, and thus

$$
\begin{aligned}
\int_{\omega_{z}}\left(|a|^{p-1}+h_{z}^{2}|\nabla f|\right)^{p^{\prime}-2} h_{z}^{4}|\nabla f|^{2} d x & \\
& \leq \int_{\omega_{z}}\left(\left|\nabla u_{h}\right|^{p-1}+h_{z}^{2}|\nabla f|\right)^{p^{\prime}-2} h_{z}^{4}|\nabla f|^{2} d x .
\end{aligned}
$$

Arguing as in the first step of the proof of Lemma 4.3 , we have

$$
\begin{aligned}
\int_{\omega_{z}} G(|a|,|\nabla v|) d x \lesssim & \int_{\omega_{z}} G\left(\left|\nabla u_{h}\right|,|\nabla v|\right) d x \\
& +\min _{K \in \mathcal{T}_{z}} \int_{\cup \mathcal{E}_{z}} G\left(\left|\nabla u_{h}\right|_{K} \mid,\left[\partial u_{h} / \partial n_{\varepsilon}\right]\right) d s .
\end{aligned}
$$

Thus the desired estimate follows from (4.31) and the above two inequalities.

Remark 4.3. It follows from the above proofs that Lemmas 4.14 .4 hold for any continuous function $G(\cdot, \cdot)$ such that it is increasing (decreasing) as $p \geq 2(p \leq 2)$ in the first argument, and is convex and increasing in the second argument.

\section{QUASI-NORM A POSTERIORI ERROR ESTIMATORS}

In this section we construct a class of a posteriori error estimators based on gradient recovery and derive a posteriori error estimates.

Definition 5.1. For any $v_{h} \in V^{h}$, let its gradient recovery $G_{h} v_{h}$ be defined by

$$
G_{h} v_{h}=\sum_{z \in D} G_{h} v_{h}(z) \varphi_{z} \quad \text { with } \quad G_{h} v_{h}(z)=\sum_{j=1}^{J_{z}} \alpha_{z}^{j}\left(\nabla v_{h}\right)_{K_{z}^{j}} .
$$

Here $\bigcup_{j=1}^{J_{z}} \bar{K}_{z}^{j}=\bar{\omega}_{z}, \varphi_{z}, \omega_{z}, D$ are defined in Definition 4.2 and $0 \leq \alpha_{z}^{j} \leq 1$, $j=1, \ldots, J_{z}$, are such that $\sum_{j=1}^{J_{z}} \alpha_{z}^{j}=1$.

Remark 5.1. There are several possible selections on $\alpha_{z}^{j}$. For example,

(1) $\alpha_{z}^{j}=\operatorname{meas}\left(K_{z}^{j}\right) / \operatorname{meas}\left(\omega_{z}\right)$. That is, $G_{h} u_{h}(z)=\int_{\omega_{z}}^{z} \nabla u_{h} / \operatorname{meas}\left(\omega_{z}\right)$.

(2) $\alpha_{z}^{j}=1 / J_{z}$. That is, $G_{h} u_{h}(z)=\left(\sum_{j=1}^{J_{z}}\left(\nabla u_{h}\right)_{K_{z}^{j}}\right) / J_{z}$.

Using the above gradient recovery, we can construct some a posteriori error estimators on any regular meshes, with upper and lower error bounds.

Theorem 5.1. Let $u$ and $u_{h}$ be the solutions of (3.1) and (3.2), respectively. Let $1<p, p^{\prime}<\infty$ with $1 / p+1 / p^{\prime}=1$ and $f \in W^{1, p^{\prime}}(\Omega)$. Then,

$$
\left|u-u_{h}\right|_{(p)}^{2} \lesssim \eta^{2}+\epsilon^{2},
$$

where

$$
\begin{aligned}
\eta^{2} & =\sum_{K \in T^{h}} \int_{K}\left(\left|\nabla u_{h}\right|+\left|\nabla u_{h}-G_{h} u_{h}\right|\right)^{p-2}\left|\nabla u_{h}-G_{h} u_{h}\right|^{2} d x, \\
\epsilon^{2} & =\sum_{K \in T^{h}} \int_{K}\left(\left|\nabla u_{h}\right|^{p-1}+h_{K}^{2}|\nabla f|\right)^{p^{\prime}-2} h_{K}^{4}|\nabla f|^{2} d x .
\end{aligned}
$$


Proof. Let $e=u-u_{h}$ and let $\pi e \in V_{0}^{h}$ be defined in Definition 4.2, It follows from (3.1), (3.2), and (3.6) that

$$
\begin{aligned}
c\left|u-u_{h}\right|_{(p)}^{2} & \leq \int_{\Omega}\left(|\nabla u|^{p-2} \nabla u-\left|\nabla u_{h}\right|^{p-2} \nabla u_{h}\right) \nabla e d x \\
& =\int_{\Omega}\left(|\nabla u|^{p-2} \nabla u-\left|\nabla u_{h}\right|^{p-2} \nabla u_{h}\right) \nabla(e-\pi e) d x \\
& =\int_{\Omega} f(e-\pi e)-\sum_{K \in T^{h}} \int_{\partial K}\left|\nabla u_{h}\right|^{p-2} \frac{\partial u_{h}}{\partial n}(e-\pi e) d x \\
& =I_{1}+I_{2} .
\end{aligned}
$$

Lemma 4.4 leads to

$$
\begin{aligned}
I_{1} \lesssim & \sum_{K \in T^{h}} \int_{K}\left(\left|\nabla u_{h}\right|^{p-1}+h_{K}^{2}|\nabla f|\right)^{p^{\prime}-2} h_{K}^{4}|\nabla f|^{2} d x \\
& +\delta_{1} \sum_{K \in T^{h}} \int_{K}\left(\left|\nabla u_{h}\right|+|\nabla e|\right)^{p-2}|\nabla e|^{2} d x \\
& +\delta_{1} \sum_{l \cap \partial \Omega=\emptyset} \min _{\bar{K} \cap l \neq \emptyset} \int_{K}\left(\left|\nabla u_{h}\right|+\left|\left[\frac{\partial u_{h}}{\partial n}\right]_{l}\right|\right)^{p-2}\left|\left[\frac{\partial u_{h}}{\partial n}\right]_{l}\right|^{2} d x \\
\lesssim & \epsilon^{2}+\delta_{1}\left(\left|u-u_{h}\right|_{(p)}^{2}\right. \\
& \left.+\sum_{l \cap \partial \Omega=\emptyset} \min _{\bar{K} \cap L \neq \emptyset} \int_{K}\left(\left|\nabla u_{h}\right|+\left|\left[\frac{\partial u_{h}}{\partial n}\right]_{l}\right|\right)^{p-2}\left|\left[\frac{\partial u_{h}}{\partial n}\right]_{l}\right|^{2} d x\right) .
\end{aligned}
$$

Define $A_{l}$ as in (3.9). With Lemmas 2.1, 2.2, and 4.3, we infer that

$$
\begin{aligned}
I_{2}= & -\sum_{K \in T^{h}} \int_{\partial K}\left|\nabla u_{h}\right|^{p-2} \frac{\partial u_{h}}{\partial n}(e-\pi e) d x \\
= & -\sum_{l \cap \partial \Omega=\emptyset} \int_{l} A_{l}(e-\pi e) d s \\
\lesssim & \sum_{l \cap \partial \Omega=\emptyset} \int_{K_{l}^{1} \cup K_{l}^{2}}\left|A_{l}\right|\left(h_{K_{l}}^{-1}|e-\pi e|+|\nabla(e-\pi e)|\right) d x \\
\lesssim & \delta_{2}^{-\beta} \sum_{l \cap \partial \Omega=\emptyset} \int_{K_{l}^{1} \cup K_{l}^{2}}\left(\left|\nabla u_{h}\right|^{p-1}+\left|A_{l}\right|\right)^{p^{\prime}-2} A_{l}^{2} d x \\
& +\delta_{2} \sum_{K \in T^{h}} \int_{K}\left(\left|\nabla u_{h}\right|+h_{K}^{-1}|e-\pi e|\right)^{p-2} h_{K}^{-2}|e-\pi e|^{2} d x \\
& +\delta_{2} \sum_{K \in T^{h}} \int_{K}\left(\left|\nabla u_{h}\right|+|\nabla(e-\pi e)|\right)^{p-2}|\nabla(e-\pi e)|^{2} d x \\
\lesssim & \delta_{2}^{-\beta} \sum_{l \cap \partial \Omega=\emptyset} \int_{K_{l}^{1} \cup K_{l}^{2}}\left(\left|\nabla u_{h}\right|^{p-1}+\left|A_{l}\right|\right)^{p^{\prime}-2} A_{l}^{2}+\delta_{2}\left|u-u_{h}\right|_{(p)}^{2} d x \\
& +\delta_{2} \sum_{l \cap \partial \Omega=\emptyset} \min _{\bar{K} \cap \neq \emptyset} \int_{K}\left(\left|\nabla u_{h}\right|+\left|\left[\frac{\partial u_{h}}{\partial n}\right]_{l}\right|\right)^{p-2}\left|\left[\frac{\partial u_{h}}{\partial n}\right]_{l}\right|^{2} d x .
\end{aligned}
$$


Noting that for all $K$ with $\bar{K} \cap l \neq \emptyset$, it holds that

$$
\begin{aligned}
\int_{K} & \left(\left|\nabla u_{h}\right|^{p-1}+\left|A_{l}\right|\right)^{p^{\prime}-2} A_{l}^{2} d x \\
= & \int_{K}\left(\left|\nabla u_{h}\right|^{p-1}+\left|\left[\left|\nabla u_{h}\right|^{p-2} \frac{\partial u_{h}}{\partial n}\right]_{l}\right|\right)^{p^{\prime}-2}\left[\left|\nabla u_{h}\right|^{p-2} \frac{\partial u_{h}}{\partial n}\right]_{l}^{2} d x \\
\leq & \int_{K}\left(\left|\nabla u_{h}\right|^{p-1}+\left|\left[\left|\nabla u_{h}\right|^{p-2} \nabla u_{h}\right]_{l}\right|\right)^{p^{\prime}-2}\left|\left[\left|\nabla u_{h}\right|^{p-2} \nabla u_{h}\right]_{l}\right|^{2} d x \\
\lesssim & \int_{K}\left(\left|\nabla u_{h}\right|^{p-1}+\left(\left|\nabla u_{h}\right|+\left|\left[\nabla u_{h}\right]_{l}\right|\right)^{p-2}\left|\left[\nabla u_{h}\right]_{l}\right|\right)^{p^{\prime}-2} \\
& \times\left(\left|\nabla u_{h}\right|+\left|\left[\nabla u_{h}\right]_{l}\right|\right)^{2(p-2)}\left|\left[\nabla u_{h}\right]_{l}\right|^{2} d x \\
= & \int_{K} Q\left(\left|\nabla u_{h}\right|+\left|\left[\nabla u_{h}\right]_{l}\right|\right)^{p-2}\left|\left[\nabla u_{h}\right]_{l}\right|^{2} d x,
\end{aligned}
$$

where

$$
\begin{aligned}
Q & :=\left(a^{p-1}+(a+b)^{p-2} b\right)^{p /-2}(a+b)^{p-2} \\
& =\left((1+b / a)^{1-p}+1 /(1+a / b)\right)^{p^{\prime}-2}
\end{aligned}
$$

and

$$
a:=\left|\nabla u_{h}\right|_{l}, b:=\left|\left[\nabla u_{h}\right]_{l}\right|
$$

It is directly verified that $Q \leq 2^{p^{\prime}-2}$ for $1<p \leq 2 \leq p^{\prime}<\infty$. When $1<p^{\prime} \leq 2 \leq$ $p<\infty$ and $a \leq b$, it holds that

$$
Q \leq(1+a / b)^{2-p^{\prime}} \leq 2^{2-p^{\prime}} .
$$

Otherwise, when $1<p^{\prime} \leq 2 \leq p<\infty$ and $a>b$,

$$
Q \leq(1+a / b)^{(1-p)\left(p^{\prime}-2\right)}=(1+b / a)^{p-2} \leq 2^{p-2} .
$$

Hence, $Q \lesssim 1$. Utilizing $Q \lesssim 1$ above, we infer that

$$
\begin{aligned}
& \int_{K_{l}^{1} \cup K_{l}^{2}}\left(\left|\nabla u_{h}\right|^{p-1}+\left|A_{l}\right|\right)^{p^{\prime}-2} A_{l}^{2} d x \\
& \quad \lesssim \int_{K_{l}^{1} \cup K_{l}^{2}}\left(\left|\nabla u_{h}\right|+\left|\left[\nabla u_{h}\right]_{l}\right|\right)^{p-2}\left|\left[\nabla u_{h}\right]_{l}\right|^{2} d x .
\end{aligned}
$$

It follows from Lemma 4.2 that

$$
\begin{aligned}
& \int_{K_{l}^{1} \cup K_{l}^{2}}\left(\left|\nabla u_{h}\right|+\left|\left[\nabla u_{h}\right]_{l}\right|\right)^{p-2}\left|\left[\nabla u_{h}\right]_{l}\right|^{2} d x \\
& \quad \lesssim \min _{K} \int_{K}\left(\left|\nabla u_{h}\right|+\left|\left[\nabla u_{h}\right]_{l}\right|\right)^{p-2}\left|\left[\nabla u_{h}\right]_{l}\right|^{2} d x .
\end{aligned}
$$


Moreover, note that $G_{h} u_{h}$ is continuous on $\Omega$. It follows from Lemma 2.1 that for any edge $l$ with $l \cap \partial \Omega=\emptyset$, we have

$$
\begin{aligned}
& \min _{\bar{K} \cap l \neq \emptyset} \int_{K}\left(\left|\nabla u_{h}\right|+\left|\left[\nabla u_{h}\right]_{l}\right|\right)^{p-2}\left|\left[\nabla u_{h}\right]_{l}\right|^{2} d x \\
= & \min _{\bar{K} \cap l \neq \emptyset} \int_{K}\left(\left|\nabla u_{h}\right|+\left|\left[\nabla u_{h}-G_{h} u_{h}\right]_{l}\right|\right)^{p-2}\left|\left[\nabla u_{h}-G_{h} u_{h}\right]_{l}\right|^{2} d x \\
\lesssim & \int_{K_{l}^{1} \cup K_{l}^{2}}\left(\left|\nabla u_{h}\right|+\left|\nabla u_{h}-G_{h} u_{h}\right|\right)^{p-2}\left|\nabla u_{h}-G_{h} u_{h}\right|^{2} d x .
\end{aligned}
$$

Then, it follows from (5.3) $-(5.4)$ that

$$
\begin{aligned}
& I_{1} \lesssim \epsilon^{2}+\delta_{1}\left(\left|u-u_{h}\right|_{(p)}^{2}+\eta^{2}\right), \\
& I_{2} \lesssim \eta^{2}+\delta_{2}\left(\left|u-u_{h}\right|_{(p)}^{2}+\eta^{2}\right) .
\end{aligned}
$$

Then (5.1) follows from (5.2), (5.5) -(5.6), and $\delta_{1}=\delta_{2}=c /(4 C)$.

Remark 5.2. The above estimates require $f \in W^{1, p^{\prime}}(\Omega)$, which may not be always true in an application. It follows from the proofs of Lemma 4.4 and Theorem 5.1 that the estimates still hold for the general case $f \in L^{p^{\prime}}(\Omega)$ as long as one replaces the term $\epsilon^{2}$ in Theorem 5.1 by

$$
\epsilon_{1}^{2}=\sum_{K \in T^{h}} \int_{K}\left(|\nabla u|^{p-1}+|f-\bar{f}| h_{K}\right)^{p^{\prime}-2} h_{K}^{2}|f-\bar{f}|^{2} d x
$$

with $\bar{f}=\int_{K} f d x / \operatorname{meas}(K)$.

Theorem 5.2. Let $u$ and $u_{h}$ be the solutions of (3.1) and (3.2), respectively. Then we have the lower bound

$$
\eta \lesssim\left|u-u_{h}\right|_{(p)}+\epsilon^{*} \quad \text { with } \epsilon^{*}=\inf _{v_{h}^{k} \in V_{h}^{k}}\left|u-v_{h}^{k}\right|_{(p)},
$$

$\eta$ defined in Theorem 5.1, the space $P_{k}$ of polynomials of the $k$-degree, and

$$
V_{h}^{k}=\left\{v_{h}^{k} \in C^{1}(\Omega): \forall K \in T^{h},\left.v_{h}^{k}\right|_{K} \in P_{k}\right\} .
$$

Proof. Set $S_{K}=\bigcup_{\bar{K}^{\prime} \cap \bar{K} \neq \emptyset} K^{\prime}$ for any $K \in T^{h}$. Note that for each $z \in D$,

$$
G_{h} u_{h}(z)=\sum_{j=1}^{J_{z}} \alpha_{z}^{j}\left(\nabla u_{h}\right)_{K_{z}^{j}}
$$

where $\sum_{j=1}^{J_{z}} \alpha_{z}^{j}=1$. Then, on the element $K$,

$$
\begin{aligned}
\left|\nabla u_{h}-G_{h} u_{h}\right| & =\left|\left(\nabla u_{h}\right)_{K}-\sum_{z \cap \bar{K} \neq \emptyset} \varphi_{z}\left(\sum_{j=1}^{J_{z}} \alpha_{z}^{j}\left(\nabla u_{h}\right)_{K_{z}^{j}}\right)\right| \\
& =\left|\sum_{z \cap \bar{K} \neq \emptyset} \varphi_{z}\left(\sum_{j=1}^{J_{z}} \alpha_{z}^{j}\left(\left(\nabla u_{h}\right)_{K}-\left(\nabla u_{h}\right)_{K_{z}^{j}}\right)\right)\right| \\
& \lesssim \sum_{K^{\prime} \subset S_{K}}\left|\left(\nabla u_{h}\right)_{K}-\left(\nabla u_{h}\right)_{K^{\prime}}\right| .
\end{aligned}
$$


Moreover, $\left|\nabla u_{h}-\sigma_{h} \nabla u_{h}\right|$ is less than or equal to a convex combination of $\mid\left(\nabla u_{h}\right)_{K}-$ $\left(\nabla u_{h}\right)_{K} \mid$. Since $\sigma(x, y)$ is monotone increasing and convex in $y$, we have

$$
\begin{aligned}
\eta^{2} & =\int_{\Omega}\left(\left|\nabla u_{h}\right|+\left|\nabla u_{h}-G_{h} u_{h}\right|\right)^{p-2}\left|\nabla u_{h}-G_{h} u_{h}\right|^{2} d x \\
& \lesssim \sum_{K \in T^{h}} \sum_{K^{\prime} \subset S_{K}} \int_{K}\left(\left|\nabla u_{h}\right|+\left|\nabla u_{h}-\left(\nabla u_{h}\right)_{K^{\prime}}\right|\right)^{p-2}\left|\nabla u_{h}-\left(\nabla u_{h}\right)_{K^{\prime}}\right|^{2} d x .
\end{aligned}
$$

It follows from Lemma 4.2 that

$$
\begin{aligned}
\eta^{2} & \lesssim \sum_{K \in T^{h}} \sum_{l \subset S_{K}} \min _{\bar{K} \cap l \neq \emptyset} \int_{K}\left(\left|\nabla u_{h}\right|+\left|\left[\nabla u_{h}\right]_{l}\right|\right)^{p-2}\left|\left[\nabla u_{h}\right]_{l}\right|^{2} d x \\
& \lesssim \sum_{l \cap \partial \Omega=\emptyset} \min _{\bar{K} \cap l \neq \emptyset} \int_{K}\left(\left|\nabla u_{h}\right|+\left|\left[\nabla u_{h}\right]_{l}\right|\right)^{p-2}\left|\left[\nabla u_{h}\right]_{l}\right|^{2} d x .
\end{aligned}
$$

It follows from Lemma 2.1 that, for any $v_{h}^{k} \in V_{h}^{k}$,

$$
\begin{aligned}
\eta^{2} & \lesssim \sum_{l \cap \partial \Omega=\emptyset} \min _{K \cap l \neq \emptyset} \int_{K}\left(\left|\nabla u_{h}\right|+\left|\left[\nabla u_{h}\right]_{l}\right|\right)^{p-2}\left|\left[\nabla u_{h}\right]_{l}\right|^{2} d x \\
& =\sum_{l \cap \partial \Omega=\emptyset} \min _{K \cap l \neq \emptyset} \int_{K}\left(\left|\nabla u_{h}\right|+\left|\left[\nabla\left(u_{h}-v_{h}^{k}\right)\right]_{l}\right|\right)^{p-2}\left|\left[\nabla\left(u_{h}-v_{h}^{k}\right)\right]_{l}\right|^{2} d x \\
& \lesssim \sum_{l \cap \partial \Omega=\emptyset} \int_{K_{l}^{1} \cup K_{l}^{2}}\left(\left|\nabla u_{h}\right|+\left|\nabla\left(u_{h}-v_{h}^{k}\right)\right|\right)^{p-2}\left|\nabla\left(u_{h}-v_{h}^{k}\right)\right|^{2} d x \\
& \lesssim \sum_{K \in T^{h}} \int_{K}\left(\left|\nabla u_{h}\right|+\left|\nabla\left(u_{h}-v_{h}^{k}\right)\right|\right)^{p-2}\left|\nabla\left(u_{h}-v_{h}^{k}\right)\right|^{2} d x .
\end{aligned}
$$

Hence

$$
\begin{aligned}
\eta^{2} \lesssim & \sum_{K \in T^{h}} \int_{K}\left(|\nabla u|+\left|\nabla\left(u_{h}-u\right)\right|\right)^{p-2}\left|\nabla\left(u_{h}-u\right)\right|^{2} d x \\
& +\sum_{K \in T^{h}} \int_{K}\left(|\nabla u|+\left|\nabla\left(v_{h}^{k}-u\right)\right|\right)^{p-2}\left|\nabla\left(v_{h}^{k}-u\right)\right|^{2} d x \\
& \lesssim\left|u-u_{h}\right|_{(p)}^{2}+\left(\epsilon^{*}\right)^{2} .
\end{aligned}
$$

Remark 5.3. By combining Theorems [5.1 and 5.2, we have that $\left|u-u_{h}\right|_{(p)}-\epsilon \lesssim$ $\eta \leq\left|u-u_{h}\right|_{(p)}+\epsilon^{*}$, where $\eta$ is the a posteriori error estimator defined in Theorem 5.1 .

$$
\epsilon^{2}=\sum_{K \in T^{h}} \int_{K}\left(\left|\nabla u_{h}\right|^{p-1}+h_{K}^{2}|\nabla f|\right)^{p^{\prime}-2} h_{K}^{4}|\nabla f|^{2} d x,
$$

and, with $V_{h}^{k}$ from Theorem 5.2, $\epsilon^{*}=\inf _{v_{h}^{k} \in V_{h}^{k}}\left|u-v_{h}^{k}\right|_{(p)}, f \in W^{1, p^{\prime}}(\Omega)$. Then,

$$
\epsilon^{2} \lesssim \begin{cases}\left(\left|u_{h}\right|_{W^{1, p}(\Omega)}^{2-p}+h^{p^{\prime}-2}|f|_{1, p^{\prime}, \Omega}^{p^{\prime}-2}\right) h^{4}|f|_{W^{1, p^{\prime}}(\Omega)}^{2} \lesssim h^{4} & \text { for } 1<p<2, \\ h^{2 p^{\prime}}|f|_{W^{1, p^{\prime}}(\Omega)}^{p^{\prime}} \lesssim h^{2 p^{\prime}} & \text { for } 2 \leq p .\end{cases}
$$

Similarly, if $u \in W^{3, p}(\Omega)$ and $v_{h}^{k}$ is the piecewise quadratic interpolation of $u$,

$$
\left(\epsilon^{*}\right)^{2} \lesssim \begin{cases}\left\|\nabla\left(u-v_{h}^{k}\right)\right\|_{L^{p}(\Omega)}^{p} \lesssim h^{2 p}|u|_{W^{3, p}(\Omega)}^{p} & \text { for } 1<p<2, \\ \left\|\nabla\left(u-v_{h}^{k}\right)\right\|_{L^{p}(\Omega)}^{2} \lesssim h^{4}|u|_{W^{3, p}(\Omega)}^{2} & \text { for } 2 \leq p .\end{cases}
$$


The conclusions above can be proved under weaker conditions. For example, it can be proved that (see [LY2])

$$
\begin{aligned}
\epsilon^{*} & =o\left(h^{2}\right) \quad \text { if } u \in W^{1+2 / p, p}(\Omega), 1<p<2 \text { or } u \in W^{2, p}(\Omega), p \geq 2, \\
\epsilon & =o\left(h^{2}\right) \quad \text { if } f \in L^{p^{\prime}}(\Omega), 1<p<2 \text { or } f \in W^{2 / p^{\prime}-1, p^{\prime}}(\Omega), p \geq 2 .
\end{aligned}
$$

Furthermore, using the results in EL, it can be shown that $\epsilon=o\left(h^{2}\right)$ and $\epsilon^{*}=$ $o\left(h^{2}\right)$ if

$$
\int_{\Omega}|\nabla u|^{p-2}\left|D^{2} u\right|^{2} d x<\infty
$$

and this condition is indeed achievable; see EL for details.

Remark 5.4. The idea used in constructing $\eta$ can be generalized to obtain new a posteriori error estimators. For example, one could define the a posteriori error estimator

$$
\left(\eta^{*}\right)^{2}=\sum_{K \in T^{h}} \int_{K}\left(\left|\nabla u_{h}\right|+\left|\nabla\left(u_{h}-u_{h}^{*}\right)\right|\right)^{p-2}\left|\nabla\left(u_{h}-u_{h}^{*}\right)\right|^{2} d x,
$$

where $u_{h}^{*}$ is the solution of a local approximation problem of (WP) as defined in the linear case $\mathrm{V2}$.

\section{ACKNOWLEDGMENTS}

The first author was partially supported by the EPSRC visiting grant GR/R28898. The work of the third author was supported by the Knowledge Innovation Program of the Chinese Academy of Sciences.

\section{REFERENCES}

[A] Adams, R.A. (1975). Sobolev Spaces. Academic Press, New York, London. MR0450957 $(56: 9247)$

[AO] Ainsworth, M. and Oden, J.T. (2000). A posteriori error estimation in finite element analysis. Wiley-Interscience [John Wiley \& Sons], New York. xx+240. MR1885308 (2003b:65001)

[BA] Baranger, J. and El Amri, H. (1991). Estimateurs a posteriori d'erreur pour le calcul adaptatif d'écoulements quasi-newtoniens. RAIRO Modél. Math. Anal. Numér., 25, 1, 31-47. MR:1086839 (91m:76070)

[BDR] Babuška, I., Durán, R. and Rodríguez, R. (1992). Analysis of the efficiency of an a posteriori error estimator for linear triangular finite elements. SIAM J. Numer. Anal., 29, 4, 947-964. MR1173179 (93d:65096)

[BL1] Barrett, J.W. and Liu, W. B. (1993). Finite element approximation of the p-Laplacian. Math. Comp., 61, 204, 523-537. MR1192966 (94c:65129)

[BL2] Barrett, J.W. and Liu, W.B. (1994). Finite element approximation of some degenerate quasi-linear problems. Lecture Notes in Mathematics 303, Pitman, 1-16. MR.1267752 (95c:65151)

[BL3] Barrett, J.W. and Liu, W.B. (1994). Quasi-norm error bounds for the finite element approximation of a non-Newtonian flow. Numer. Math., 68, 4, 437-456. MR1301740 (95h:65078)

[BS] Brenner, S.C. and Scott, L.R. (1994). The mathematical theory of finite element methods. Springer Verlag, New York, 15, xii+294. MR1278258 (95f:65001)

[Ca] Carstensen, C. (1999). Quasi-interpolation and a posteriori error analysis in finite element method. M2AN Math. Model. Numer. Anal., 33, 6, 1187-1202. MR1736895 (2001a:65135)

[CB] Carstensen, C. and Bartels, S. (2004). Averaging techniques yield reliable a posteriori finite element error control for obstacle problems. Numer. Math. 99 225-249. MR2107431 
[CF1] Carstensen, C. and Funken, S.A. (2000)a. Constants in Clement-interpolation error and residual based a posteriori estimates in finite element methods. East-West J. Numer. Math., 8, 3, 153-175. MR1807259 (2002a:65173)

[CF2] C. Carstensen and S. A. Funken. Averaging technique for FE-a posteriori error control in elasticity. Part I: Conforming FEM. Comput. Methods Appl. Mech. Engrg., 190 (2001) 2483-2498. Part II: $\lambda$-independent estimates. Comput. Methods Appl. Mech. Engrg., 190 (2001) 4663-4675. Part III: Locking-free conconforming FEM. Comput. Methods Appl. Mech. Engrg., 191 (2001), no. 8-10, 861-877. MR.1815651|(2002a:74114) MR1840795 (2002d:65140) MR:1870519(2002j:65106)

[CK] Carstensen, C. and Klose, R. (2003). Guaranteed a posteriori finite element error control for the p-Laplace problem. SIAM J. Scientific Computing 25 792-814. MR2046112 (2005d:65203)

[Ch] Chow, S.-S. (1989). Finite element error estimates for nonlinear elliptic equations of monotone type. Numer. Math., 54, 4, 373-393. MR0972416 (90a:65235)

[Ci] Ciarlet, P.G. (1978). The Finite Element Method for Elliptic Problems. North-Holland Publishing Co., Amsterdam, xix+530. MR0520174 (58:25001)

[EL] Ebmeyer, C. and Liu, W.B. Quasi-norm interpolation error estimates for finite element approximations of problems with p-structure. (to appear).

[GM] Glowinski, R. and Marrocco, A. (1975). Sur l'approximation, par éléments finis d'ordre un, et la résolution, par pénalisation-dualité, d'une classe de problèmes de Dirichlet non linéaires. Rev. Francaise Automat. Informat. Recherche Opérationnelle Sér. Rouge Anal. Numér., 9, R-2, 41-76. MR0388811 (52:9645)

[KJF] Kufner, A., John, O. and Fučík, S. (1977). Function spaces. Noordhoff International Publishing, Leyden, xv+454. MR0482102 (58:2189)

[L] Lieberman, G.M. (1988). Boundary regularity for solutions of degenerate elliptic equations. Nonlinear Anal., 12, 11, 1203-1219. MR0969499 (90a:35098)

[LB5] Liu, W. B. and Barrett, J.W. (1996). Finite element approximation of some degenerate monotone quasilinear elliptic systems. SIAM J. Numer. Anal., 33, 1, 88-106. MR.1377245 (97j:65180)

[LY1] Liu, W. and Yan, N. (2001). Quasi-norm local error estimators for p-Laplacian. SIAM J. Numer. Anal., 39, 1, 100-127. MR.1860718 (2002i:65131)

[LY2] Liu, W.B. and Yan, N. (2001). Quasi-norm a posteriori error estimates for nonconforming FEM of p-Laplacian. Numer. Math., 89, 341-378. MR1855829(2002g:65136)

[LY3] Liu, W. and Yan, N. (2001). Some a posteriori error estimators for p-Laplacian based on residual estimation or gradient recovery. J. Sci. Comput., 16, 4, 435-477 (2002). MR:1881854 (2002k:65191)

[ODSD] Oden, J. T., Demkowicz, L., Strouboulis, T. and Devloo, P. (1986). Adaptive methods for problems in solid and fluid mechanics. (Accuracy estimates and adaptive refinements in finite element computations (Lisbon, 1984)), Wiley Ser. Numer. Methods Engrg., Wiley, Chichester, 249-280. MR0879450 (88d:73010)

[P] Padra, C. (1997). A posteriori error estimators for nonconforming approximation of some quasi-Newtonian flows. SIAM J. Numer. Anal., 34, 4, 1600-1615. MR1461798 (98h:65050)

[V1] Verfürth, R. (1994). A posteriori error estimates for nonlinear problems. (Finite element discretizations of elliptic equations), Math. Comp., 62, 206, 445-475. MR.1213837 (94j:65136)

[V2] Verfürth, R. (1996). A review of a posteriori error estimation and adaptive meshrefinement techniques, Wiley-Teubner. MR.1643051

Humboldt-Universität zu Berlin, Unter den Linden 6, 10099 Berlin, Germany

E-mail address: cc@math.hu-berlin.de

CBS \& Institute of Mathematics and Statistics, University of Kent, Canterbury, CT2 7NF, ENGLAND

E-mail address: W.B.Liu@ukc.ac.uk

Institute of System Sciences, Academy of Mathematics and System Sciences, Chinese Academy of Sciences, Beijing, People's Republic of China

E-mail address: yan@amss.ac.cn 